\title{
Cartographie des linéaments géologiques en domaine aride par extraction semi-automatique à partir d'images satellitaires: Exemple à la région d'EI Kseïbat (Sahara algérien)
}

\author{
Geological lineament mapping in arid area by semi-automatic \\ extraction from satellite images: example at the El Kseïbat \\ region (Algerian Sahara)
}

\author{
N. Hammad, M. Djidel, N. Maabedi \\ Université KasdiMerbah Ouargla, Laboratoire de Géologie du Sahara, Faculté des Hydrocarbures, des Energies \\ Renouvelables et des Sciences de la Terre et de l'Univers, Ouargla 30000, Algérie. Email: hyperspectre007@yahoo.fr
}

\section{RÉSUMÉ}

Les géologues en charge d'une cartographie des linéaments détaillée en domaine aride et désertique, font face à l'étendue des terrains et la présence abondante de dépôts éoliens. La présente étude présente une approche semi-automatique différente des autres méthodes d'extraction des linéaments, à savoir l'extraction automatique et l'extraction manuelle par le fait d'être à la fois rapide et objective. Elle consiste en une succession de traitements numériques (filtrage textural et spatial, binarisation par seuillage et morphologie mathématique...etc.) appliqués sur une scène LANDSAT-7 ETM+. Cette approche semi-automatique a permis d'obtenir une carte détaillée des linéaments, tout en tenant compte des directions tectoniques reconnues dans la région. Elle permet d'atténuer l'effet des dépôts dunaires répondant aux spécifications du contexte aride. La validation visuelle de ces structures linéaires, effectuée sur terrain et sur la base de la carte géologique et des données géoscientifiques existantes, a permis l'identification de la majorité des linéaments jugés structuraux ou du moins géologiques.

Mots clés: linéaments; cartographie; semi-automatique; filtrage; domaine aride.

\section{ABSTRACT}

Geologists in charge of a detailed lineament mapping in arid and desert area, face the extent of the land and the abundance of eolian deposits. This study presents a semi-automatic approach of extraction of lineament, different from other methods, such as the automatic extraction and manual extraction, by being both fast and objective. It consists of a series of digital processing (textural and spatial filtering, binarization by thresholding and mathematic morphology ... etc.) applied to a Landsat 7 ETM+scene. This semi-automatic approach has produced a detailed map of lineaments, while taking account of tectonic directions recognized in the region. It helps mitigate the effect of dune deposits meet the specifications of arid environment. The visual validation of these linear structures, by geoscientists and field data, allowed the identification of the majority of structural lineaments or at least those tried geological.

Keywords: lineaments; mapping; filtering; semi-automatic; arid area.

Citation / Cómo citar este artículo: Hammad, N. et al. (2016). Cartographie des linéaments géologiques en domaine aride par extraction semi-automatique à partir d'images satellitaires: Exemple à la région d'El Kseïbat (Sahara algérien). Estudios Geológicos 72(1), e049. http://dx.doi.org/10.3989/egeol.42158.377.

Copyright: (C) 2016 CSIC. This is an open-access article distributed under the terms of the Creative Commons Attribution License (CC BY) Spain 3.0. 


\section{Introduction}

Dans une image, les éléments linéaires sont représentés par tous les objets filiformes tels que routes et rivières, pour ceux qui sont mieux définis mais également par les limites de parcelles telles que haies, talus, etc. (Hobbs, 1912; Lattman, 1958; O'Leary et al., 1976; Chorowicz \& Mukonki, 1979; Chorowicz et al., 1989).

Les linéaments qui nous intéressent, nous géologues, sont ceux d'origine naturelle et qui correspondent le plus souvent à des lignes de crêtes topographiques, des contacts entre formations de lithologies différentes, des ensembles de plis et des lignes de fractures ou de failles (Pélissonnier, 1974; Nicolini, 1980; Scanvic, 1993; Ozer, 1989).

Ils peuvent être identifiés visuellement par leur effet sur le paysage (Lachaine, 1999). D'autres linéaments dont la signification géologique est probable ou douteuse (Fourcade, 1979) peuvent être reconnus telle la géométrie des rives d'un plan d'eau ou la forme très rectiligne du réseau de drainage, des anomalies de végétation qui pourraient correspondre à des anomalies géochimiques du sous-sol (Lefèvre, 1979) ou à des structures profondes (Tonelli, 1975). Scanvic, (1986), constate que les structures géologiques contrôlent tellement la morphologie de la surface terrestre que, dans presque tous les cas, les linéaments extraits d'une image satellitaire caractérisent ces structures à un très fort degré.

L'extraction de ces éléments linéaires s'effectue généralement suivant deux approches: par photointerprétation ou automatiquement (Caloz \& Colet, 2001; Provencher \& Dubois, 2007; Sukumar et al., 2014).

La photo-interprétation continue à être la démarche habituelle utilisée pour de multiples travaux de cartographie détaillée dans différents domaines (Lattman,1958; Joshi 1989; Lillesand \& Kiefer, 1994; Jourda et al., 2006; Provencher \& Dubois, 2007; Kouamé et al., 2009). Cependant elle présente l'inconvénient d'être subjective et laborieuse (Moore, 1986; Joshi, 1989; Dubois, 1999).

L'extraction automatique est effectuée grâce à de nombreux algorithmes avancés (Moore et Waltz, 1983; Canny, 1986; Burns et al., 1986; Toumazet, 1990; Masuda et al., 1991; De sève et al., 1994; Argialas et al., 2000; Caloz \& Collet, 2001; Hung et al., 2005; Hanifi, 2009; Abdullah et al., 2010;
Mahdi \& Jawad, 2015) qui parviennent à détecter les linéaments et à les extraire. Bien que rapide et objective, cette approche présente le désavantage de ressortir tous les éléments linéaires net ou à bord net présents dans l'image sans tenir compte de leur nature (naturel ou anthropologique).

Compte tenu des avantages et inconvénients de chacune de ces approches jugées inadéquates à nos conditions de terrain, une approche semiautomatique est proposée.

En effet le contexte d'étude caractérisé par sa grande étendue, sa complexité structurale et l'abondance des dépôts dunaires rend nécessaire une nouvelle manière d'extraction qui à travers une succession de traitements et de filtrages permet de pallier ces contraintes.

\section{Cadre géologique de l'étude}

La région d'El Kseïbat connue sous le nom de Gourara se situe dans la partie nord-ouest du Sahara algérien, sur le territoire de la wilaya d'Adrar.

A l'intersection des principaux éléments structuraux du sud-ouest algérien (Figure 1), le secteur d'étude constitue à la fois la partie centrale de la zone de jonction entre la chaîne de l'Ougarta et la région de bled El Mass (bordure occidentale du bassin de l'Ahnet) (Wilckzinsky et al., 1987) et la zone de suture de l'extrémité orientale du craton ouest africain d'âge éburnéen et la partie externe de la

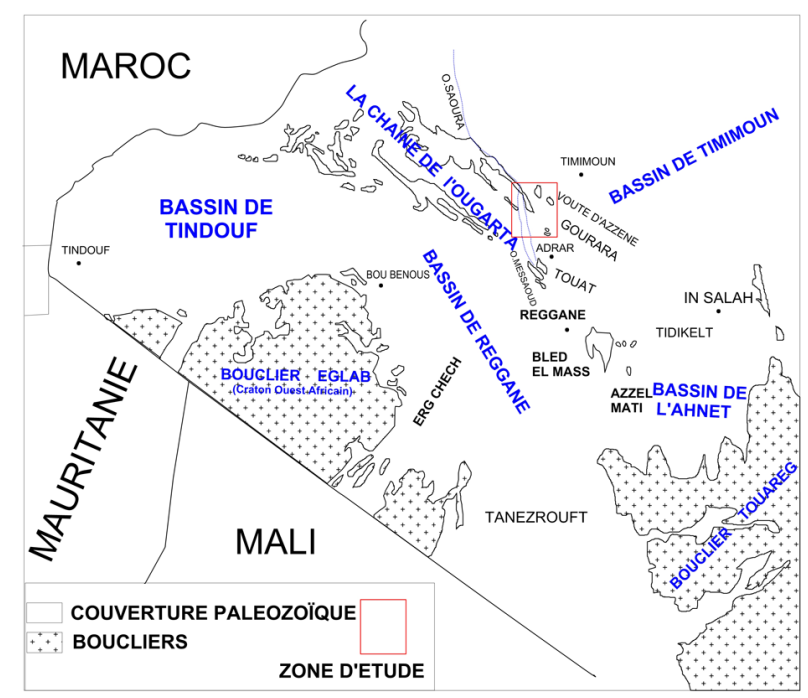

Fig. 1.-Contexte géologique régional de la zone d'étude. 
plateforme panafricaine. Géologiquement, la région est limitée au nord-est, par la voûte d'Azzène qui la sépare du bassin de Timimoun et au sud-ouest, par la chaîne de l'Ougarta qui la sépare du bassin de Reggane au niveau de la zone haute de Bled El Mass. Vers le Sud-Est elle est séparée du bassin de l'Ahnet par l'ensellement de l'Azzel-Matti.

Le substratum sur lequel s'est déposée la série sédimentaire n'a pas été caractérisé du fait qu'aucun affleurement n'a été décrit dans la région. Sa description s'est effectuée essentiellement à la lumière des données de subsurface (forage, sismique, gravimétrie et aéromagnétisme) (Wilckzinsky et al., 1987; Drid, 1989).

Les principales formations sédimentaires décrites dans cette région appartiennent au paléozoïque, affleurant dans sa partie septentrionale (Figure 2), surmontées en discordance de séries méso-Cénozoïques qui recouvrent la partie méridionale. Cette discordance témoigne du plissement varisque de la région.

La région se caractérise également par un grand développement de sédiments crétacés et quaternaires et par la présence de nombreux paléochenaux plio-quaternaires (Bensefia, 2005).

Le contexte structural de la région a été difficile à décrire en raison de sa situation sur la zone qui limite le Craton Ouest Africain et la Plateforme Saharienne et à la terminaison sud-est de la chaine hercynienne de l'Ougarta.

Les mouvements tectoniques verticaux et horizontaux manifestés durant les différentes étapes de l'histoire

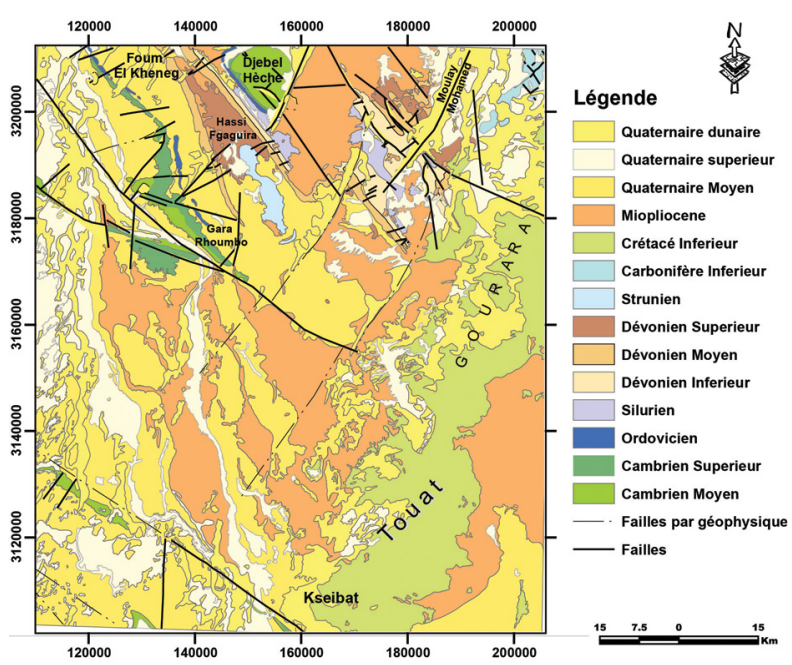

Fig. 2.-Carte géologique d'El Kseibat (Wilckzynski et al., 1987 modifiée). géologique, essentiellement paléozoïques, sont à l' origine des nombreuses structures et failles sculptées sur le schéma structural de la région (Figure 3)

\section{Données disponibles}

Notre étude s'appuie sur image extraite d'une scène LANDSAT 7 ETM+, p197r 40, acquise le 16/04/2000 (Figure 4). Ces images satellitaires ont été choisies du fait de leur caractéristique spectrale et spatiale permettant une bonne cartographie structurale à petite échelle (Scanvic, 1993; Jourda et al., 2006). Les traitements sont effectués sur le logiciel ENVI 4.5.

L'image ETM+ utilisée apparait sans bruit radiométrique majeur et ne nécessite donc pas de traitement radiométrique. Par contre une correction géométrique de type "image à carte" a été effectuée afin de les rendre parfaitement superposables aux cartes thématiques existantes (cartes topographiques, géologiques). A partir de douze couples de points d'amer, bien distribués, relevé sur l'image ETM+ et sur la carte géologique au $1 / 200000^{\text {ème }}$, les coordonnées originales (ligne et colonne) sont traitées par ordinateur pour obtenir les nouvelles coordonnées au sol.

De plus nous disposons de cartes géologiques régionales au $1 / 500000^{\mathrm{eme}}$ couvrant les régions de Kerzaz, Timimoune et Adrar, d'une carte géologique d'El Kseïbat au $1 / 200000^{\text {ème }}$ établie par Wilckzinsky et al., (1987). Une mission sur terrain a été effectuée en janvier 2014 pour la validation des résultats. Pour valider certains linéaments non reconnus sur les travaux de surface, l'étude s'est appuyée également sur des rapports inédits des études géophysiques à savoir gravimétriques et aéromagnétiques réalisées par la Sonatrach (1986) et l'Aéroservice (1975) respectivement.

\section{Méthodologie}

La méthodologie adoptée pour la cartographie des linéaments s'est effectuée en deux étapes successives et complémentaires.

Une première étape d'amélioration et renforcement pour faciliter l'extraction de l'information relative aux linéaments repérés dans l'image grâce aux frontières linéaires entre deux zones de brillance différente ou de texture différente (Nguyen \& Ho, 1988; Fraipont \& Hirsch, 1984; Lachaine, 1999; Caloz \& Collet, 2001). 


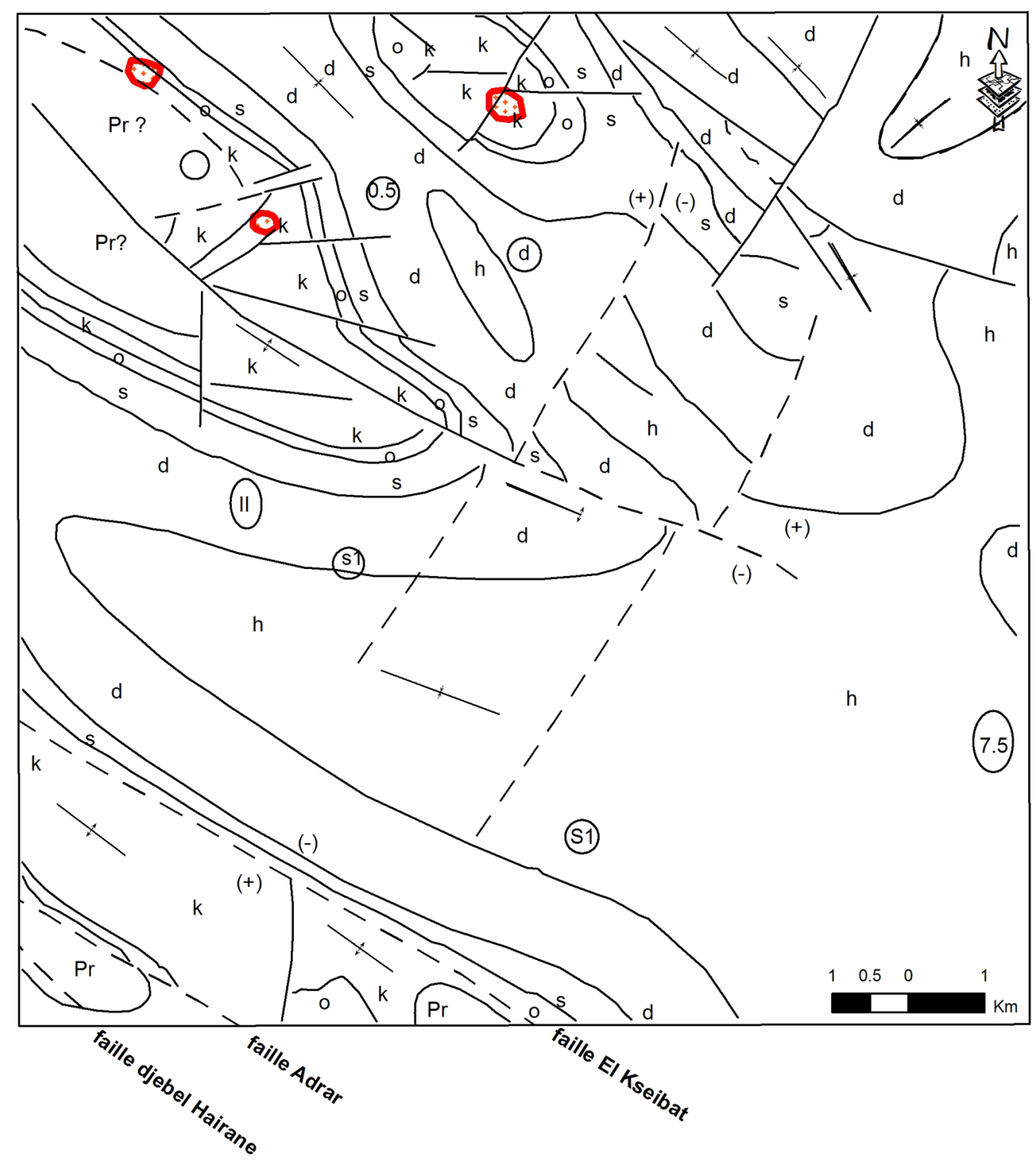

\section{Légende}

\begin{tabular}{|c|c|c|c|c|}
\hline 0 & ordovicien & $\mathrm{h}$ & Carbonifère & $\begin{array}{ll}\text { _- limite stratigraphique }(+) \text { bloc soulevé } \\
\text { (-) bloc affaissé }\end{array}$ \\
\hline k & cambrien & d & dévonien & $\longrightarrow$ axe anticlinal \\
\hline $\mathrm{Pr}$ & protérozoïque & $\mathrm{s}$ & silurien & - - faille géophysique (1) \\
\hline
\end{tabular}

Fig. 3.-Schéma structural de la région. 


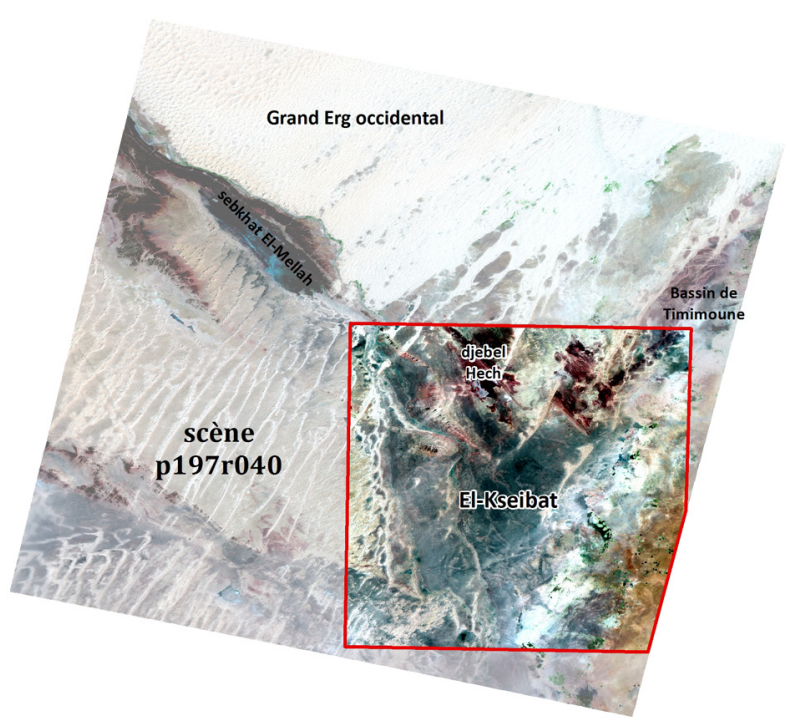

Fig. 4.-Localisation du secteur dans la scène p197r40ETM+de LANDSAT.
La deuxième partie est l'extraction des linéaments proprement dite. Le schéma ci-dessous (Figure 5) résume la méthodologie de travail réalisée. Les filtres appliqués et les différentes opérations effectuées sont donnés par ordre de réalisation.

\section{Filtrage et Traitements de rehaussement \\ Traitement de transformation}

Les plus couramment utilisés parmi les traitements de transformation (Scanvic, 1993; Jourda et al., 2006) d'image sont l'Analyse en Composantes Principales (ACP) et les ratios.

L'analyse en composante principale est une technique efficace pour accentuer une image multispectrale à des fins d'interprétation géologique. Elle consiste en une transformation mathématique (Bonn \& Rochon,

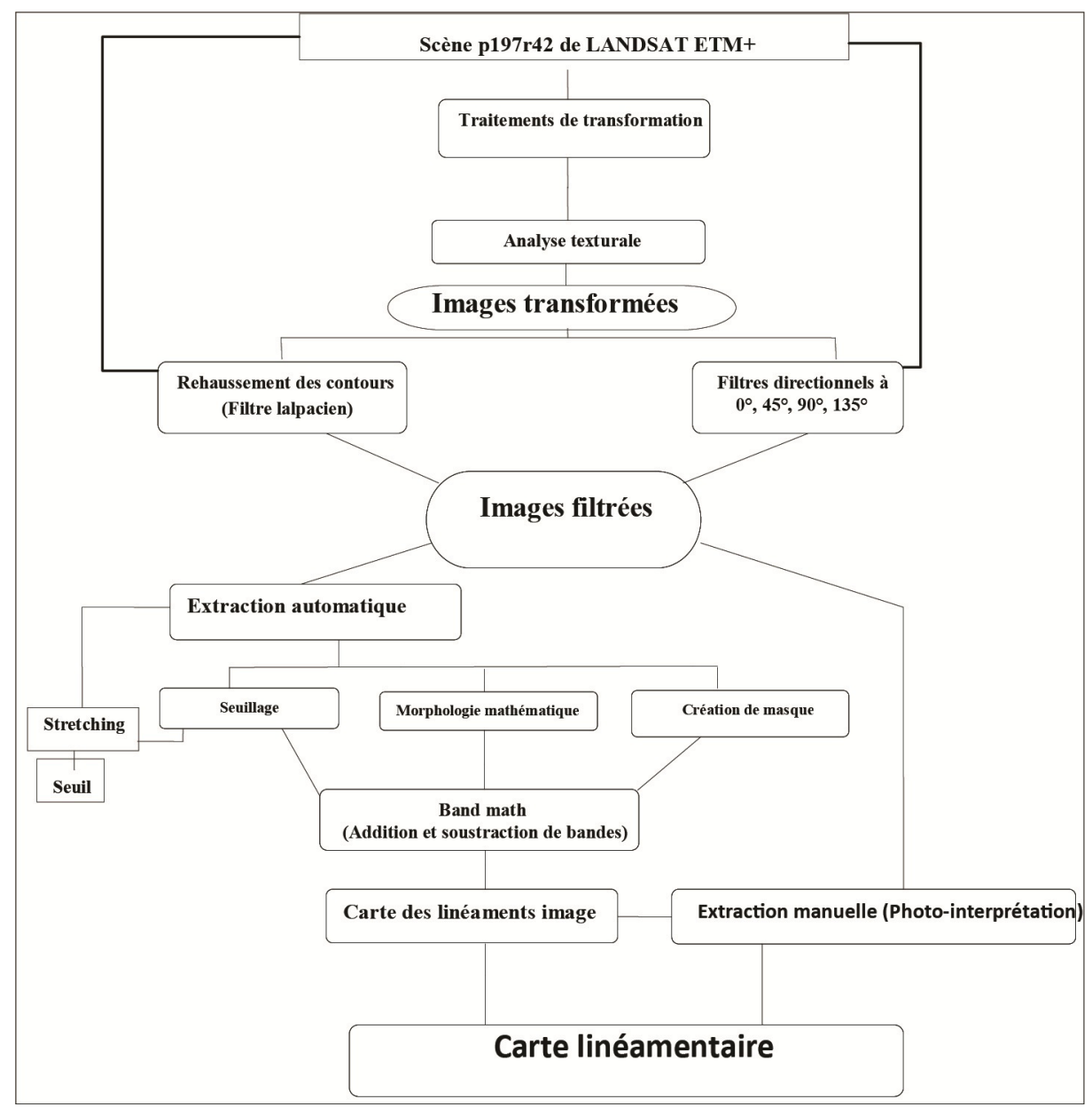

Fig. 5.-Organigramme des traitements effectués. 
1992; Jourda et al., 2006) qui génère des nouvelles images, composantes ou axes, qui sont en combinaison linéaire avec les images originales. Les trois premières composantes contiennent un grand pourcentage de la variance totale présente dans les six bandes originales des canaux ETM+. Le ratios ou calcul des rapports spectraux est une transformation d'image très commune. Il permet de renforcer et rehausser des variations subtiles dans les réponses spectrales de différents objets et de rehausser certains détails contenus dans l'image (Youan et al., 2008). Pour notre étude, seule l'atténuation des effets d'ombrage liés au relief a été réalisée, grâce aux rapports de bandes ETM+3/ $\mathrm{ETM}+4, \mathrm{ETM}+4 / \mathrm{ETM}+5$ et $\mathrm{ETM}+4 / \mathrm{ETM}+7$.

\section{L'analyse texturale}

L'information texturale dans une image est donnée par la forme et la configuration d'un groupe de pixel de niveau de gris différents (Lake, 1991). La méthode développée par Haralick (1979) est une méthode statistique qui permet de sélectionner les paramètres qui définissent le mieux les éléments structurants en mesurant la distribution des tons de gris par des matrices de cooccurrence pour déterminer les relations entre un pixel donné et son voisinage (Kouamé et al., 1999; Kouamé et al., 2009). Cette matrice de cooccurrence, appréciée dans une multitude de travaux visant la caractérisation, classification et la modélisation des textures (Lohmann, 1995; Caloz et Colet, 2001; Hanifi, 2009), est appliqué sur les bandes ETM3 et ETM7. Ces deux bandes sont sélectionnées pour leur fiabilité en analyse structurale et en discrimination lithologique (Scanvic, 1993) due à leur sensibilité aux moindres variations des tons de gris dans l'image engendrée par des variations lithologiques.

Huit indices de cooccurrence normalisés (Figure 6) sont calculés et créés à partir de l'ensemble de la matrice mais seulement trois indices ont été retenu homogénéité, dissemblance et moyenne qui accentuent les principaux éléments structuraux (Gotlieb \& Kreyszig, 1990; Kouamé et al., 1999; Hanifi, 2009).

\section{Filtrage spatiale des images}

Les filtres spatiaux (Tableau 1) sont des opérateurs locaux qui selon Caloz et Collet (2001) prennent en compte le voisinage d'un élément du signal, du pixel en traitement d'images, pour accentuer, atténuer ou extraire une propriété locale telle que le gradient, la moyenne, ou d'autres paramètres statistiques. Leur application permet d'épurer l'image de manière que les lignes frontières se présentent plus nettement sans toutefois réduire l'information àl'intérieur des unités spatiales en d'autres termes la détection et le renforcement des contours.

Comme il est question d'accentuer et de dégager les linéaments correpondant à des discontinuités lithologiques ou structurales, nous avons opté au départ pour un filtrage linéaire par des opérateurs de convolution, type laplacien, spécialement établi pour détecter et rehausser les contours. Cepandant, ces filtres présentent l'inconvénient de dédoubler les lignes de contour (Caloz et Colet, 2001). Pour cela on lui a préfèré d'autres filtres directionnels. Ces derniers améliorent la perception des linéaments en provoquant un effet optique d'ombre porté sur l'image comme si elle était éclairée par une lumière rasante (Marion, 1987) et permettent de rehausser les linéaments qui ne sont pas favorisés par la source d'éclairement (Drury, 1986). Sous ENVI 4.5, ce filtrage a été établi dans plusieurs directions $\left(0^{\circ}, 45^{\circ}, 90^{\circ}\right.$ et $\left.135^{\circ}\right)$ avec une fenêtre de $5 \times 5$ pour les linéaments majeurs et $3 \times 3$ pour les structures plus fines (Figure 7). Les bandes utilisées pour les filtrage directionnel sont les bandes ETM3, ETM7, les ratios $(\mathrm{ETM}+3 / \mathrm{ETM}+4$, $\mathrm{ETM}+4 / \mathrm{ETM}+7$ ) et l'ACP1.

L'ensemble des images résultant du filtrage directionnel ainsi que celles des indices texturaux d'homogénéité, de dissemblance et de moyenne ont toutes été retenues pour l'étape suivante de l'extraction des linéaments.

\section{Extraction des linéaments}

\section{Binarisation par seuillage des images}

La phase qui permet réellement d'obtenir le contour est la biarisation. Après transformation et filtrage, l'image est en ton de gris et le contour n'est obtenu qu'après avoir binarisé l'image transformée (Dubois,1999). Le choix du seuil de binarisation est très délicat et pose deux problèmes. Si l'on applique un seuil trop bas certains linéaments ne pourront plus ètre discernés dans la masse de conours retenus (micro-contours, faux 


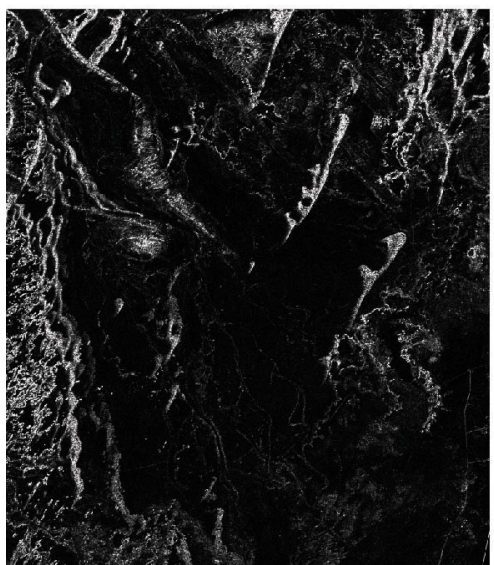

Indice de variance

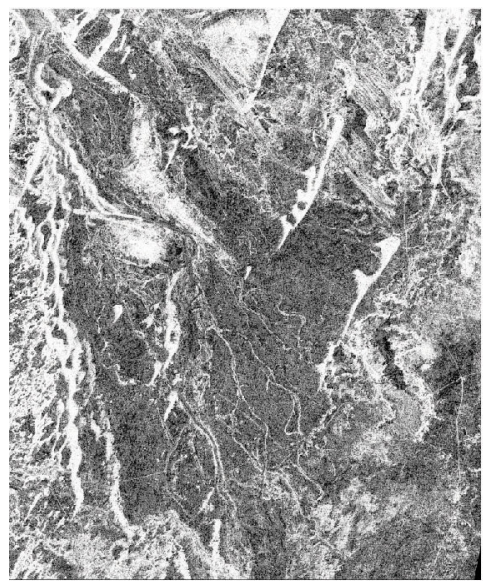

Indice de dissimilarité

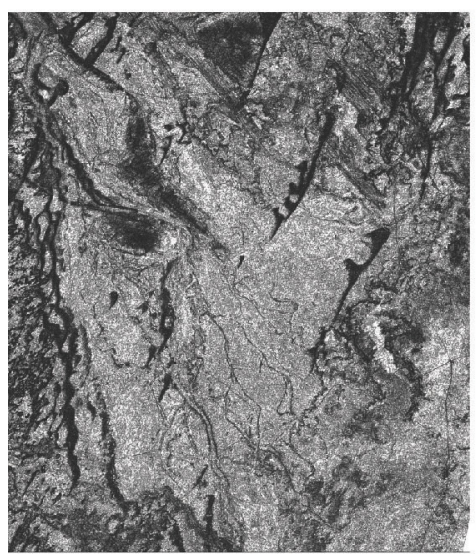

Indice de second moment

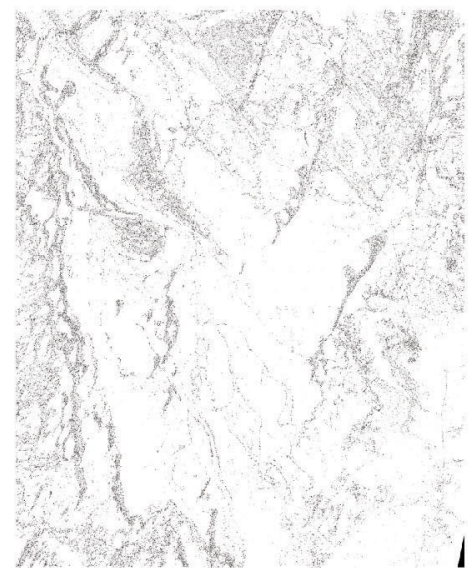

Indice de corrélation

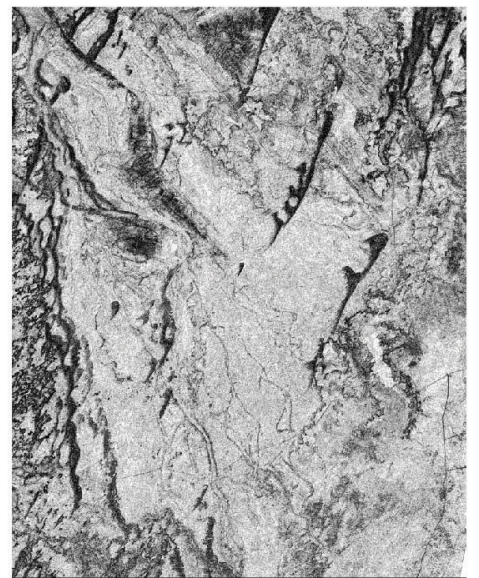

Indice d'homogeneité

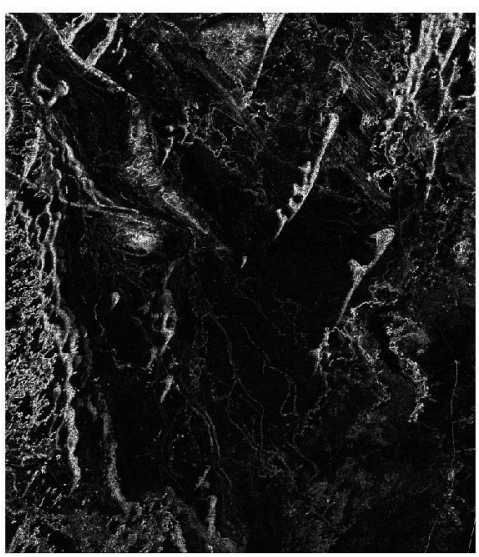

Indice d'entropie

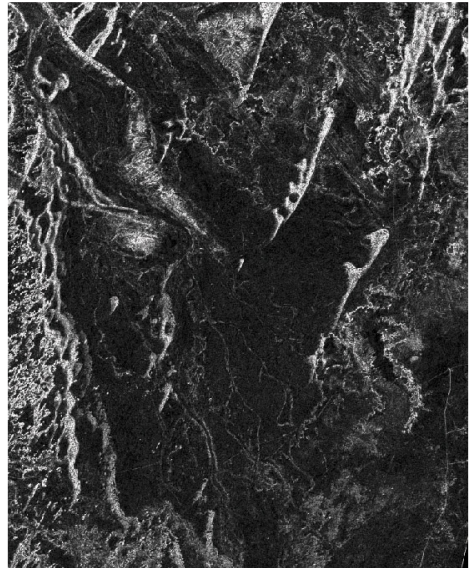

Indice de contraste

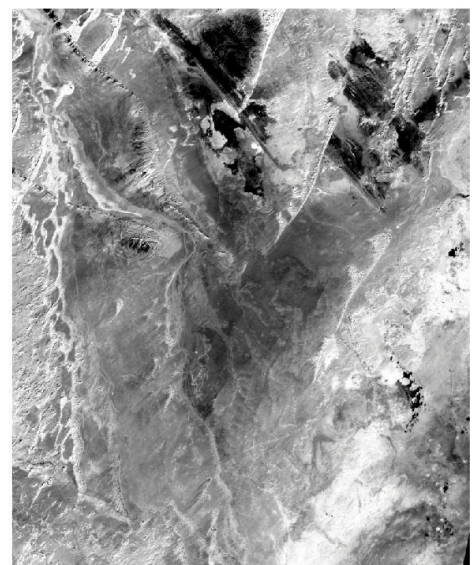

Indice de Moyenne

Fig. 6.-Les indices de cooccurrence.

contours et zones hétérogènes). Si l'on retient un seuil trop élevé les linéaments deviennent discontinus et certains disparaissents ou ne sont plus que de rares points. Pour l'estimation du seuil approprié nous avons eu recour à l'histogramme des fréquences par un streching interactif. Un seuil acceptable fut déterminé manipulant les extrémités de l'histogramme de fréquence. 
Tableau 1.-Les opérateurs de filtres spatiaux (Caloz \& Collet, 2001).

\begin{tabular}{llll}
\hline Fonction & Opérateur de convolution & Opérateur statistique & Opérateur morphologique \\
\hline Lissage (passe-bas) & Moyenne mobile simple & Moyenne & Erosion \\
& Moyenne mobile pondérée & Médiane & Dilatation \\
& & Mode & Ouverture \\
& & & Fermeture \\
& & & Squeletisation \\
Rehausssement local (passe-haut) & Gradient omnidirectionnel (lalpacien) & Amplitude & Gradient morphologique \\
& Gradient directionnel (Sobel, Prewit) & Ecart type & Chapeau haut-de-forme \\
& & Quantile & Fond de valée \\
\hline
\end{tabular}
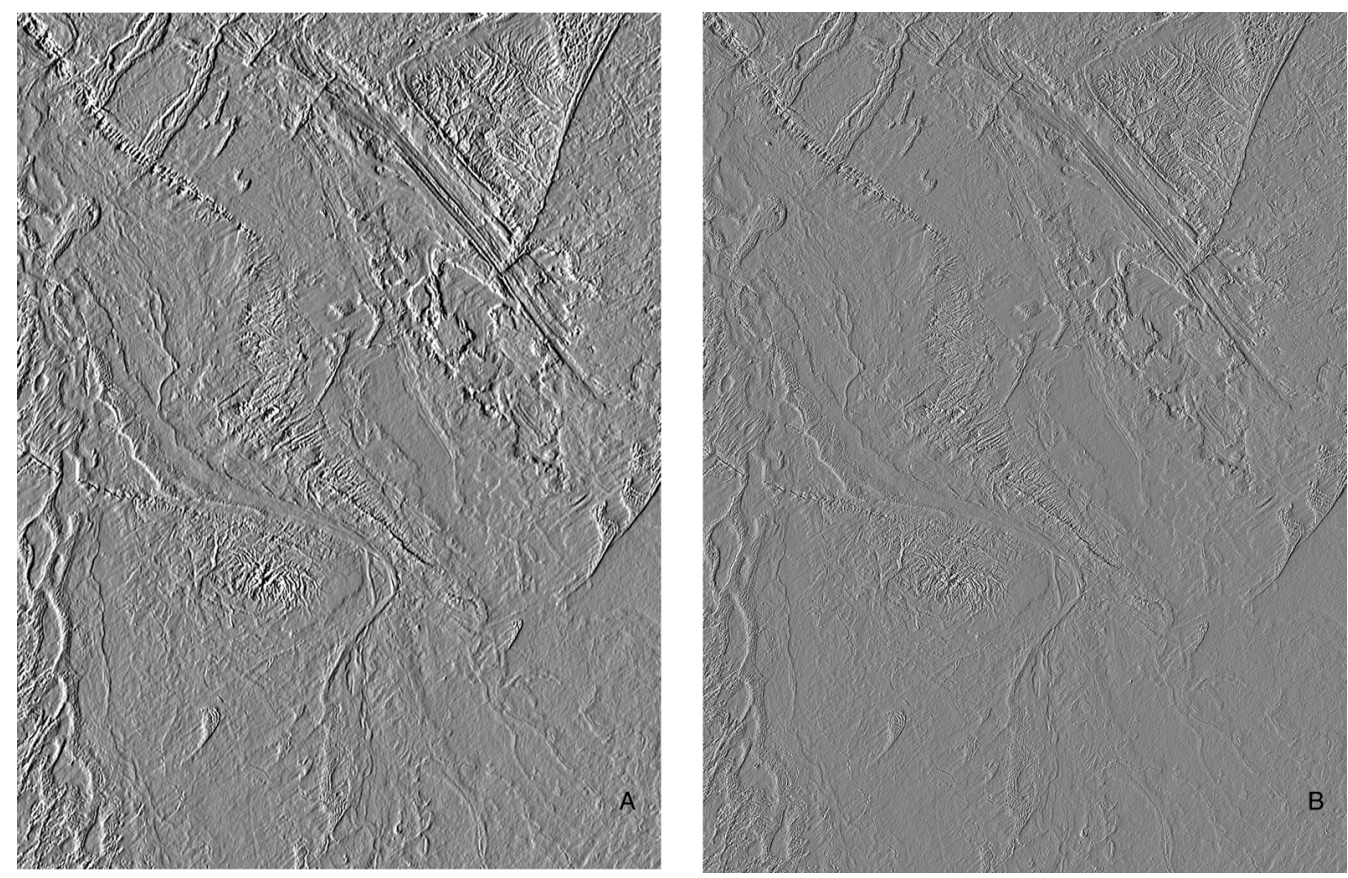

Fig. 7.-Extraits du filtre directionnel à $45^{\circ}$ appliqués sur la bande ETM3: A) fenêtres 5x5, B) fenêtre $3 \times 3$.

\section{Morphologie mathématique}

Les images issues de l'étape précédente ressortent bien les éléments structuraux contenus dans l'image mais restent toujours remplies de bruit et de pixels libres entre les linéaments. Pour y remédier un opérateur morphologique d'érosion qui vise à amincir ou squeletiser les contours (Bonn et Rochon, 1992 ; Kouamé et al., 1999; Caloz et Colet, 2001) a été appliqué. Après il était nécéssaire de reconnecter les parties où le contour se perd, en utilisant un autre opérateur morphologique, la dilatation. Nous avons été amené à répéter les opérations érosion/ dilatation pour bien amincir les contours sans les rendre discontinus. Pour éliminer certaines tâches composées de pixels sans pour autant modifier la taille des autres objets nous avons appliqué une opération d'ouverture qui consiste en une érosion suivie d'une dilatation (Caloz et Colet, 2001). Une opération de fermeture visant l'élimination des petits trous au milieu des contours sans modifier leur taille, termine cette procédure de filtrage morphologique.

Les images résultantes sont de bonne qualité visuelle ressortent les eléments linéaires contenu dans l'image. Ces images ressortaient également les contours des abondantes formations dunaires couvrant la région.

Dans les étapes suivantes, il est question de rassembler l'information contenue dans l'ensembles des bandes et de diminuer l'effet du sable qui ressortait sur les images filtrées et seuillées. 


\section{Extraction des linéaments}

Pour regrouper l'information contenue sur les différentes images et obtenir une image omnidirectionnelle nous avons tout simplement additionné les images binarisées (filtres texturaux retenus et filtres directionnels) par la fonction band math. La nouvelle image ainsi obtenue de cette sommation aura des valeurs comprises entre ( 0 ) et (n) selon la valeur du pixel et le nombre de bandes aditionnées (n).

L'image obtenue est par la suite binarisée par l'application d'un masque qui réduit à 1 les valeurs superieures à 1 et maintient les pixels à valeur 0 et 1 .

Sur cette image seuls les contours des sables et des cordons dunaires, très abondants, posaient problème en se confondent avec certains linéaments géologiques. Pour épurer l'image de ces fourmillants contours et petits segments sans avoir à les suprimer manuellement, nous avons procédé par la création d'un masque des dunes (Figure 8). Créée par une classification supervisée par la méthode support vector Machine (SVM) (Hanifi, 2009), la classe dune est transformée en un vecteur qui a servi à la construction du masque. Cette étape a permis le seuillage des dunes qui seront codées sur 1 et le reste sera codé sur 0 . Cette image

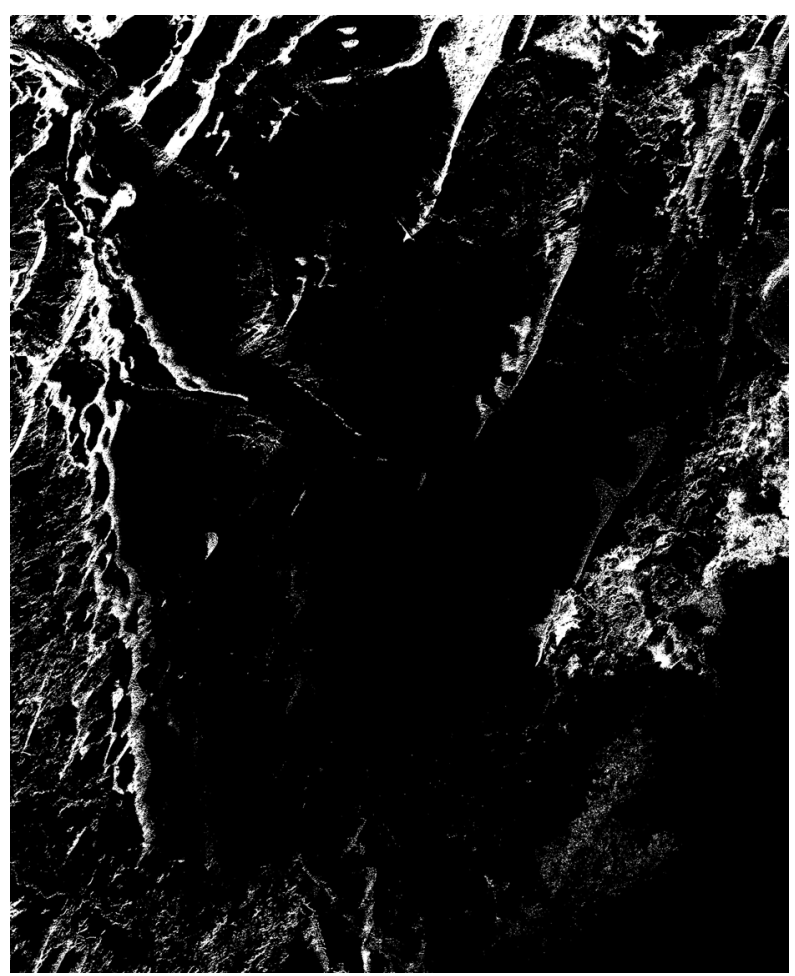

Fig. 8.-Masque de la classe dune. est par la suite soustraite par band Math de l'image binarisée des linéaments. Tout les pixels à valeur 1 correspondant à des sables sont soustraits de l'image seuilée et leur valeur devient alors 0 .

\section{Résultats et Discussion}

La technique d'extraction semi-automatique proposée s'avère assez rapide et objective. Le choix d'un enchainement cohérent et logique de traitements atténuant les différentes contraintes du terrain constitue l'étape la plus délicate parla détection des seuils les plus appropriés et l'application des masques adéquats (masque de la classe dune). La particularité réside dans le faite d'avoir additionnées les images filtrées et seuillées pour obtenir les linéaments existant dans l'ensemble des directions décrites. De plus, les fourmillants segments correspondant aux crêtes et aux contours des dunes ont été éliminés par la soustraction du masque de la formation dunaire.

La validation du résultat final présentés sur la carte des linéaments (Figure 9) est effectuée visuellement par confrontation aux différents documents géoscientifiques disponibles (carte géologique et

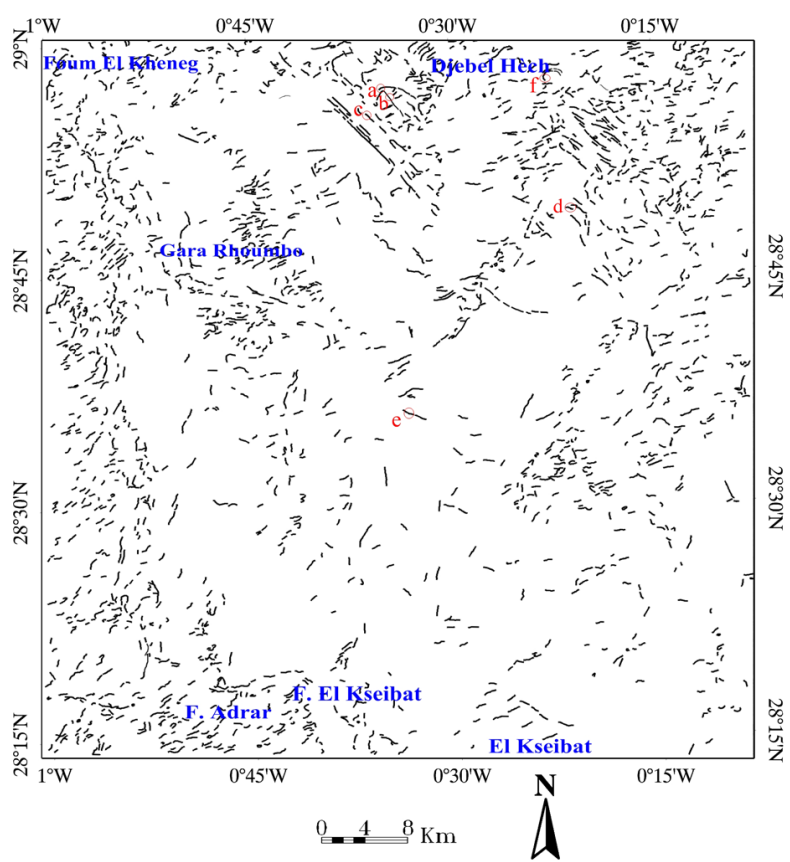

Fig. 9.-Carte des linéaments géologiques de la région d'el kseibat (a, b, c, d, e voir figure 10). 
données géophysiques) et sur terrain. Le résultat est assez satisfaisant car la carte obtenue ressort tous les accidents reconnus par les études antérieurs aussi bien de surface que de subsurface:

- Les linéaments orientés NO-SE (direction ougartienne) et NE-SO correspondent bien à des accidents tectoniques.

- Les deux grands linéaments observés sur la partie méridionale de l'image, également validés par données géophysiques, correspondent aux accidents d'El Kseibat, et de Adrar décrits dans les travaux de Wilckzinsky et al. (1987).

- Les linéaments correspondant à des lits d'oueds ont bien été identifiés grâce à leur morphologie typique.
Certains linéaments non mentionnés sur les travaux antérieurs ont été vérifié sur terrains (Figure 10):

Les linéaments observés au Djebel Hèch correspondent à des zones de mylonitisation associées à de nombreuses minéralisations (Figure 10a et b), d'autres à un fauchage ou redressement des couches plissées $(\mathrm{c}, \mathrm{d})$ ou à certains bancs compétents (calcaires à orthoceres du silurien).

- Les linéaments curvilignes observés dans la partie centrale de la région (Figure 9) correspondent à des paléo-chenaux plio-quaternaires dont les talus sont jalonnés de grands blocs de grès cambriens (Figure 10e).

- Des linéaments géomorphologiques (Figure 10f) ont également été retenus et correspondent à des
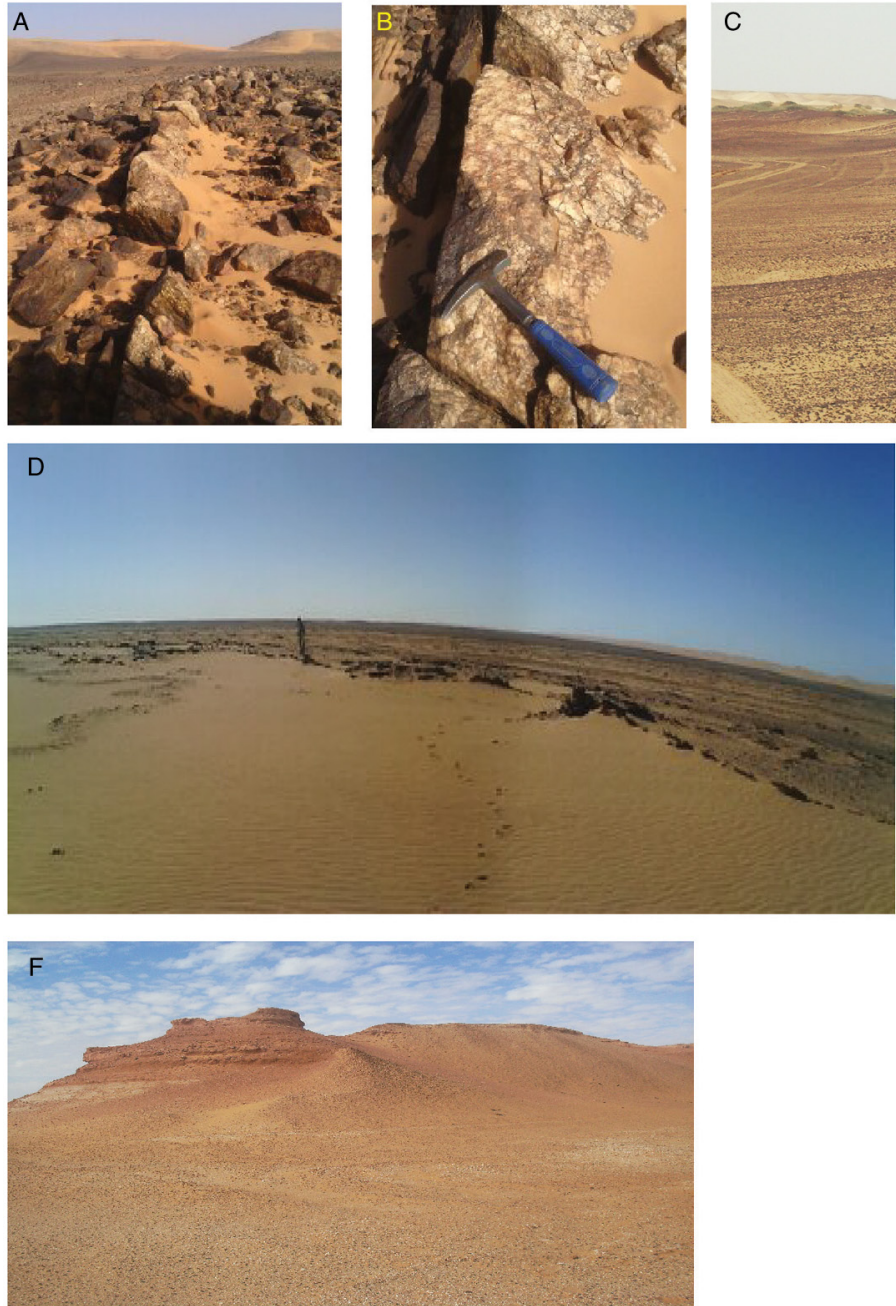
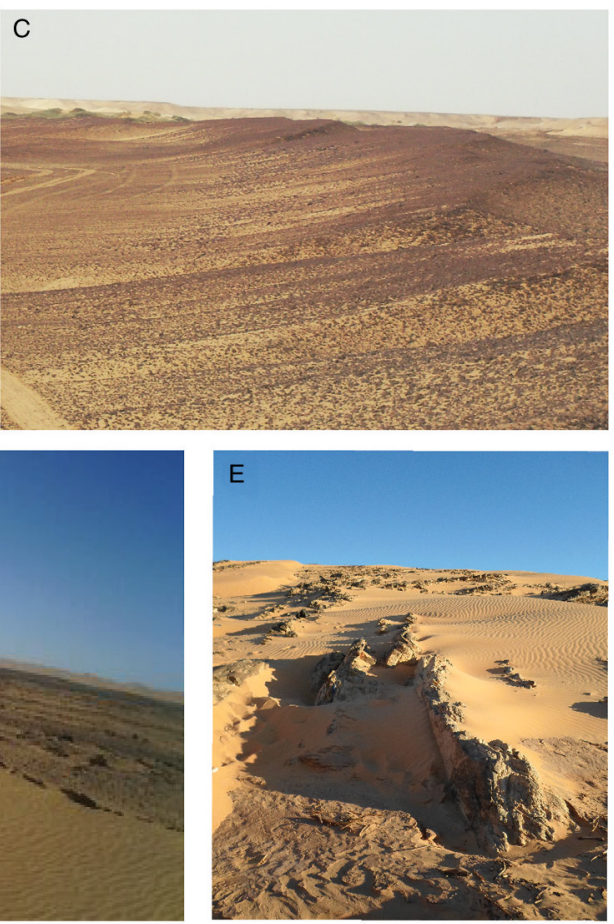

Fig. 10.-Photos des différents linéaments vérifiés sur terrain (situation voir figure 9). 
buts témoins mio-pliocènes mais ne seront pas pris en compte pour l'interprétation structurale prévue ultérieurement.

L'image des discontinuités-image ressort également de nombreuses structures circulaires et demi circulaires dont certaines (à l'Est de Djebel Hèch et Gara Roumbo) ont été préalablement identifiée par des données géophysiques (Wilckzinsky et al., 1987) comme étant des corps magmatiques non affleurant (à environ 100m de profondeur). D'autres structures circulaires, induites par la morphologie des sables, ressortant sur la partie occidentale de l'image n'ont pas été identifiée en raison de l'inaccessibilité de cette partie du terrain.

Nous jugeons que les résultats sont satisfaisants comparés à d'autres auteurs qui considèrent que l'extraction semi-automatique ne leur a pas fourni les résultats prévus (Kouamé et al., 1999) en raison de la difficulté à sélectionner les paramètres qui caractérisent les discontinuités image.

\section{Conclusion}

L'extraction semi-automatique des discontinuités images est une approche qui présente l'avantage d'être pratique et rapide qui allie l'automatisme de l'ordinateure et le raisonnement de l'analyste. Elle répond bien au besoin urgent d'établir une carte détaillée des discontinuités image et de dresser une base de données des linéaments dans une région en contexte aride. Intégrée dans un système d'information géographique (Shupe \& Akha, 1989), la carte des linéaments obtenue permet la mise à jour des données déjà existantes et l'adjonction de nouveaux segments pouvant êtrelié à la répartition des multiples minéralisations décrites dans la région (Wilckzinsky et al., 1987).

\section{REMERCIEMENT}

Les auteurs tiennent à remercier chaleureusement Mme Danielle Ducrot pour son accueil chaleureux durant leur stage effectué au sein Centre d'Etudes Spatiales de la Biosphère (CESBIO, Toulouse, France) et ses précieux conseils et éclaicissements.

\section{Réferences}

Aeroservice coorporation (1975). Aero-magneto-spectrometric Survey of Algeria: Final report. 3 volumes, Houston, Philadelphia.
Abdullah, A.; Akhir, J.M. \& Abdullah, I. (2010). The extraction of lineaments using slope image derived from digital elevation model: Case study of Sungai Lembing - Maran area, Malaysia. Journal of Applied Sciences Research, 6: 1745-1751.

Argialas, D.; Mavrantza, O. \& Stefouli, M. (2000). Automatic mapping of tectonic lineaments (fault) using methods and techniques of photo interpretation/ digital remote sensing and expert systems. THALES Project, 1174, 6 pp.

Bensefia, K.E. (2005). Contribution à l'étude des apports sédimentaires d'une zone clés des bassins tertiaire quaternaire de l'axe Adrar-Reggane (exemple: la région d'el Kseibet). Thèse magistère, Université Tlemcen, Algerie, 124 pp.

Bonn, F. \& Rochon, G. (1992). Précis de télédétection, Volume 1. Presses de l'université du Québec, Québec, 485 pp.

Burns, J.B.; Hanson, A.R. \& Risemean, E.M. (1986). Extracting straight lines. IEEE Transactions on Pattern Analysis and Machine Intelligence, 8 (4): 425-455. http://dx.doi.org/10.1109/TPAMI.1986.4767808

Caloz, R. \& Collet, C. (2001). Précis de télédétection, Volume 3 : Traitements numériques d'images de télédétection. Presses de l'université du Québec, Québec, 398 pp.

Canny, J.F. (1986). A computational approach to edge detection. IEEE Transactions on Pattern Analysis and Machine Intelligence, 8 (6): 679-698. http:// dx.doi.org/10.1109/TPAMI.1986.4767851

Chorowicz J.; Cervelle B.; Kim J.; Manossis S.; Morasse C.; Prudon D.; Rudant J.P.; Riazanoff S.; Breard J.Y.; Foin P. \& Veillet I. (1989). Utilisation des modèles numériques de terrain pour la reconnaissance automatique de certaines formes en géologie et géomorphologie. Bulletin - Société française de photogrammétrie et de télédétection, 115 (3): 5-7.

Chorowicz, J. \& Mukonki M. (1979). Linéaments anciens. Zones transformantes récentes et géotectonique de fossés de l'Est africain d'après la Télédétection et la microtectonique. Musée Royal de l'Afrique Central, Département de Géologie et Mines, Rapport Annuel 1979: 143-167.

De Sève, D.; Desjardins, R. \& Toutin, T. (1994). Contribution de données radar d'ERS-1 dans l'appréhension de l'organisation des linéaments: le cas de l'astroblème de Charlevoix. Journal canadien de télédétection, 20 (3): 233-244.

Drid, M. (1989). Sur quelques aspects de la diagenèse organique et minérale dans le bassin de Timimoune et le sillon de Sbaa (Sahara central algérien).Thèse Doctoral, Université de bordeaux, 245pp.

Drury, S.A. (1986). Image Interpretation in Geology. Allen \& Unwin, London, 243 pp.

Dubois, J. (1999). Identification des linéaments dans les images satellitaires par ajustement et suivi de segment. Mémoire de Maîtrise, Université de Sherbrooke, 123 pp. 
Fourcade B. (1979). Deux méthodes d'utilisation de la télédétection en géologie (cartographie automatique des éléments linéaires, stéréoscopie gravimétrique).Thèse du 3ème cycle, Université de Bordeaux III, 231 pp.

Fraipont, P. \& Hirsch, J. (1984). Analyse linéamentaire: procédure de traitement de données télédétectés. Computers in earth sciences for natural resources characterization: colloque international, Nancy, France, avril 1984.

Gotlieb C.C. \& Kreyszig H.E. (1990). Texture descriptors based on co-occurrence matrices. Computer Vision, Graphics, and Image Processing, 51 (1): 70-86. http://dx.doi.org/10.1016/S0734-189X(05)80063-5

Hanifi, M. (2009). Extraction de caractéristiques de texture pour la classification d'images satellites. Thèse, Université Toulouse III, Paul Sabatier, 97 pp. http://thesesups.ups-tlse.fr/675/

Haralick, R. M. (1979). Statistical and structural approaches to texture. Proceedings of the IEEE, 67 (5): 786804. http://dx.doi.org/10.1109/PROC.1979.11328

Hobbs, W.H. (1912). Earth features and their meaning: an introduction to geology for the student and the general reader. Macmillan, New-York, 506 pp.

Hung, L.Q.; Batelaan, O. \& De Smedt, F. (2005). Lineament extraction and analysis, comparison of LANDSAT ETM and ASTER imagery. Case study: Suoimuoi tropical karst catchment, Vietnam. Remote Sensing for Environmental Monitoring, GIS Applications and Geology V, Proc. of SPIE, 5983, 12pp. http://dx.doi.org/10.1117/12.627699

Joshi, A.K. (1989). Automatic detection of lineaments from LANDSAT Data. IGARSS 89: $12^{\mathrm{e}}$ Symposium Canadien sur la Télédétection. Volume 1: $85-88$.

Jourda, J.P.; Djagoua, E.V.; Kouamé, K.F.; Saley, M.B.; Gronayes, C.; Achy, J.J.; Biémi, J. \& Razack, M. (2006). Identification et cartographie des unités lithologiques et des accidents structuraux majeurs du département de Korhogo (Nord de la Côte d'Ivoire): Apport de l'imagerie ETM+ de Landsat. Revue Télédétection, 6 (2): 123-142.

Kouamé, F.; Gioan, P.; Biemi, J. \& Affian K. (1999). Méthodes de cartographie des discontinuités -images extraites d'images satellitales: exemple de la région semi-montagneuse à l'ouest de la côte d'Ivoire. Revue de Télédétection, 1 (2): 139-156.

Kouamé, F.; Lasm, T.; Saley, M.B.; Tonyé, E.; Bernier, M. \& Wade, S. (2009). Extraction linéamentaire par morphologie mathématique sur une image RSO de RadarSat-1: application au socle Archéen de la Côte d'Ivoire. IIIèmes Journées d'Animation Scientifique du réseau de Télédétection de l'AUF, Alger, 8-11 novembre 2009.

Lachaine, G. (1999). Structures géologiques et linéaments, Beauce (Québec). Mémoire de maîtrise, Département de géographie et télédétection, Université de Sherbrooke, 83 pp.
Lake, L.A. (1991). Analyse des méthodes texturales appliquées au traitement des images. Département de Géographie et télédétection. Université de Sherbrooke, $38 \mathrm{pp}$.

Lattman, L.H. (1958). Technique of mapping geologic fracture traces and lineaments on aerial photographs. Photogrammetric Engineering, 24: 568-576.

Lefevre, M.J. (1979). Détection aéroportée de phénomènes géologiques de sub-surfaces révélées par une anomalie de température de brillance (Coat en Noz, Côtes du Nord) France. Revue photo interprétation, 6 (3a).

Lillesand, T.M. \& Kiefer, R.W. (1994). Remote sensing and image interpretation. 3 ed. John Wiley and Sons, New York, $750 \mathrm{pp}$.

Lohmann, G. (1995). Analysis and synthesis of textures: a co-occurrence-based approach. Computers \& Graphics, 19: 29-36. http://dx.doi. org/10.1016/0097-8493(94)00119-J

Mahdi, A.S. \& Jawad, L.A. (2015). An Adaptive Automatic Algorithm for Extracting Geological Lineaments in Al-Dibdibba Formation Basin. Iraqi Journal of Science, 56 (1C): 866-874.

Marion, A. (1987). Introduction aux techniques de traitement d'image. Eyrolles, Paris, 278 pp.

Masuda, Sh.; Tokuo, T.; Ichinose, T.; Otani, K. \& Uchi, T. (1991). Expert system for lineament extraction from Optical Sensor Data. Geoinformatics, 2 (2): 195-200.

Moore, G. (1986). Digital processing of Landsat-TM for lineament occurrence and spatial frequency in sedimentary Rock. U.S. Department of interior, Geological Survey Report DOE, OR21552-1, EROS Data Center.

Moore G.K. \& Waltz F.A. (1983). Objective procedure for lineament enhancement and extraction. Photogrammetric Engineering \& Remote Sensing, 49 (5): 641-647.

Nguyen, P.T. \& Ho, D. (1988). Multiple source data processing in remote sensing. In: Digital Image Processing in Remote Sensing (Muller, J.P., Ed.), Taylor and Francis, Philadelphia, Pa, USA, 153-176.

Nicolini , P. (1980). Méthodes de la Gîtologie. Ed. M. Deschamps, Haïti. 218 pp.

O'Leary, D.W.; Friedman, J.D. \& Pohn, H.A. (1976). Lineament, linear, lineation: Some proposed new standards for old terms. Geological Society of America Bulletin, 87: 1463-1469. http://dx.doi. org/10.1130/0016-7606(1976)87<1463:LLLSPN $>2$. $0 . \mathrm{CO} ; 2$

Ozer, A. (1989). Ressources minérales et ressources en eau: l'apport de la télédétection dans le monde francophone. In: Dubois, J.M. (Ed.). Télédétection en francophonie. AUPELF-UREF, John Libbey Eurotext, 117-123.

Pelissonnier, H. (1974). Vers une métallogénie quantitative. Annales des Mines, Mars 1974: 47-58. 
Provencher, L. \& Dubois, J.M. (2007). La photointerprétation au Québec: Historique et perspectives. Télédétection, 6 (1): 57-70.

Scanvic, J.-Y. (1986). Quelques aspects géobotaniques et structuraux de la télédétection spatiale : implications dans la prospection minière du massif armoricain, France. First European workshop on Remote Sensing in mineral exploration. Report EUR 10511, Commission of the European Communities, 127-147.

Scanvic, J.-Y. (1983). Utilisation de la télédétection dans les sciences de la Terre. Bureau de recherches géologiques et minières, Orléans, $159 \mathrm{pp}$. Manuels et méthodes, 7.

Scanvic, J.-Y. (1993). Télédétection aérospatiale et informations géologiques. Bureau de recherches géologiques et minières, Orléans, $284 \mathrm{pp}$.

Shupe, S.M. \& Akha, V. (1989). Integration of remotely sensed and G.I.S. data for mineral exploration: Halifax Pluton area, Nova Scotia, Canada. Geocarto International, 4 (3): 49-54. http://dx.doi. org/10.1080/10106048909354220

Sonatrach (1986). Synthèse gravimétrique de l'AhnetMouydir - Gourara. Rapport interne inédit. Division Hydrocarbures, Alger, $15 \mathrm{pp}$.

Sukumar, M.; Venkatesan, N. \& Nelson Kennedy Babu, C. (2014). A Review of Various Lineament
Detection Techniques for high resolution Satellite Images. International Journal of Advanced Research in Computer Science and Software Engineering, 4: $72-78$.

Suzen, M.L. \& Toprak, V. (1998). Filtering of satellite images in geological lineament analyses: an application to a fault zone in Central Turkey. International Journal of Remote Sensing, 19 (6): 1101-1114. http://dx.doi.org/10.1080/014311698215621

Tonelli, A. M. (1975). Contribution of ETRS-1 and Skylab missions to regional studies in Italy. Journal of the British Interplanetary Society, 28 : 647-652.

Toumazet, J.J. (1990). Le traitement d'image par l'exemple. Editions Sybex, 260 pp.

Wilckzinsky, M.; Pizon, A.; Pokorski, J.; Juskou, O. \& Moscicki, W. (1987). Travaux de levé et de recherche systématique au 1/200000 sur la feuille NH-30-VI El KSEIBAT, Rapport inédit SONAREM, 109 pp.

Youan Ta, M.; Lasm, T.; Jourda, J. P.; Kouamé, K. F. \& Razack, M. (2008). Cartographie des accidents géologiques par imagerie satellitaire Landsat-7 ETM+ et analyse des réseaux de fractures du socle précambrien de la région de Bondoukou (nord est de la Côte d'Ivoire), Télédétection, 8(2): 119-135. 